all other ways of doing direct heart massage, as it involves less risk of hæmorrhage, trauma, and shock, and can subsequently be more quickly and satisfactorily closed.

We are indebted to Lieutenant-Colonel Bond for permission to publish this case.

References.-1. T. A. Green : The Lancet, 1906, ii., 1708-1714. See also Cack ovic: : Archiv

2. Frazier: Journal Amer. Med. Assoc., 1911, 1vi., 1448. Lambert: Austral. Med. Journal, 1911-12, N.S. i. 527, Wrede : Arch klin Chir., 1913, ci., 833. Weitz : Deutsch. med. Woch., 1915, xli., 338 . Breitman Trans. Internat. Med. Congress, 1913, Sec. v., Pt. 2, 264. A. R. Thomas Journal Roy. Nav. Med. Service, 1918, iv. 91.

3. W. M. Mollison : Brit. Journal Child. Dis., 1917, xiv., 42.

\section{A MODIFICATION OF}

\section{WEBSTER'S TEST FOR THE PRESENCE OF T.N.T. IN URINE.}

\section{BY FRANK TUTIN.}

(From the Department of Bioohemistry and Pharmaoology, Medical Research Committee.)

IT has been shown by Webster ${ }^{1}$ that T.N.T., when taken by the mouth, or otherwise absorbed into the system, is excreted in an altered form in the urine, where its presence may readily be detected. The urine to be examined is first extracted with ether, then acidified with a mineral acid and again extracted with ether. In the latter extract the presence of the azoxy-compound formed by metabolisation from T.N.T. is readily shown by the development of a violet tint on the addition of alcoholic potash.

\section{Possible Source of Error.}

There is one possibility of confusion which, though it has probably been recognised by a number of workers, has apparently not hitherto been mentioned in published literature. Various purgatives (rhubarb, senna, cascara, \&c.) contain substances (anthraquinone derivatives) which, when excreted in the urine, give under the conditions of Webster's test a red or reddish-violet colour, which might be mistaken for that produced by T.N.T. It is well known in medical practice that the urine of patients taking purgatives of this group will directly give a red colour with alkali, and this is rather loosely attributed to "chrysophanic acid." Chrysophanic acid is only one, and not the most important, of the substances concerned, and it seemed desirable to investigate the whole group from the point of view of their excretion and colour-producing properties, and to devise some modification of the T.N.T. test which would eliminate the possibility of confusion.

The results of this short investigation form the subject of this note. When I had completed it and had found that a slight modification of Webster's test made possible a clear distinction between the colour reaction due to T.N.T. and that due to any of the anthraquinone derivatives occurring in purgatives, I heard from Mr. Webster that he had himself come across the same possibility of error, that he had adopted the same modification of his test as that which I here suggest, and that he had found, like myself, that its introduction in any case sharpened and improved the T.N.T. reaction, whether anthraquinone derivatives were present or not.

My experiments were made firstly with the pure anthraquinone derivatives which occur in the different purgatives of this class, and secondly with doses of rhubarb root, since this drug contains all the known substituted anthraquinones present in the group.

The pure principles in question are rhein, aloe-emodin, emodin," and chrysophanol ("chrysophanic acid"). They are all closely related chemically, the relation being briefly as follows: chrysophanol is a methyldihydroxyanthraquinone, aloe-emodin is the corresponding hydroxymethyldihydroxyanthraquinone, rhein the carboxylic acid yielded by the oxidation of aloe-emodin, and emodin is chrysophanol containing one additional, strongly phenolic

\section{The Lancet, 1916, ii., p. 1029.}

2 Fmodin monomethyl ether, another constituent of rhubarb, was not separately investigated, since this substance always behaves exactly similarly to chrysophanol, unless it suffers demethylation, when, of course, it yields emodin. hydroxyl group. These substances were each administered by the mouth in $0.1 \mathrm{~g}$. doses, and in each case the urine subsequently collected, when examined by Webster's method, gave a strong colour reaction which might easily have been mistaken for that produced by T.N.T. On further investigating these four samples of urine it was found that the rhein had been rapidly excreted unchanged, whilst the other three anthraquinone derivatives had each undergone more or less oxidation, the aloe-emodin being entirely, and the chrysophanol partially, converted into the corresponding carboxylic acid (rhein), whilst a portion of the emodin appeared to be oxidised to the corresponding trihydroxyanthraquinone carboxylic acid, a substance which has not hitherto been known. In all cases, however, it was found that an ethereal liquid could be obtained which yielded no colour with alcobolic potash if the extracts obtained from the acidified urine were washed with dilute aqueous sodium carbonate before being treated with the stronger alkali. It was, moreover, found that the azoxy-compound formed from T.N.T., which is the substance which yields the characteristic "Webster reaction," is not removed from ether by treatment with dilute aqueous sodium carbonate.

\section{Desoription of Modified Test.}

These results, therefore, indicate a method by which anthraquinone derivatives may be eliminated from the ethereal extract to be tested by Webster's method, and it is recommended that the test should be conducted in the following modified manner :-

The urine should first be extracted with two successive portions of ether. This treatment removes any T.N.T. which may be present from accidental contamination, and also chrysophanol. The urine is then acidified with hydrochloric or sulphuric acid ana again extracted with ether. During these extractions the addition of alcohol is usually necessary to cause the separation of emulsions. The ethereal extract of the acidified uxine must then be extracted twice with dilute aqueous sodium carbonate, washed with water, and then treated with alcoholic potash, when the characteristic violet colour will be produced if derivatives of T.N.T. were present in the urine. If anthraquinone derivatives were present the sodium carbonate extracts will have: a reddish -violet colour.

The method as originally described by Webster, and the modified procedure given above were then applied to samples of urine from three people who had previously taken respectively (a) 0.01 g. T.N.T., (b) 0.01 g. T.N.T. $+1 \mathrm{~g}$. rhubarb root, and (c) $1 \mathrm{~g}$. rhubarb root. When following Webster's procedure all three samples gave a positive reaction, but after treatment with sodium carbonate $(a)$ and (b) gave reactions due to T.N.T. only, whilst (c) gave no reaction. Moreover, the colour yielded by $(a)$ after treatment with sodium carbonate was a much purer violet and appeared more intense than before this treatment. This was due to the fact that sodium carbonate removes from the ethereal extract small amounts of substances which give a yellow or brownish colour with alkali, and therefore mask, to some extent, the characteristic colour of the "Webster reaction." The employment of the modified test here proposed would therefore appear to be desirable on all occasions when examining urine for T.N.T., even when the possibility of the presence of anthraquinone derivative is known to be precluded.

In conclusion, the author wishes to express his indebted. ness to the Wellcome Chemical Research Laboratories, who supplied the specimens of pure anthraquinone derivatives which have been mentioned.

THE death is announced of Miss Agnes E. Weston, G.B.E., the "Sailor's Friend," at the Royal Sailor's Rest, Devonport, at the age of 78 .

KING'S Cortiage HosptTal Medical SCHOOL (UNIVERSTY OF IONDON). The University Entrance Scholarship in Anatomy and Physiology (value $£ 50$ ) has been awarded to Miss A. M. Freeman.

The Nation's Tribute To NuRses.-A meeting in support of the Hon. Sir Arthur Stanley's appeal to the nation for $\$ 100,000$ as a tribute fund for nurses was held in Belfast on Oct. 17th. It was addressed, among others, by Mr. Henry McLoughlin, of Dublin, organising secretary of the movement in Ireland. The money raised in Ireland is to be invested in four Dublin trustees, and the interest is to be used on behalf of Irish nurses. It was decided to appeal for funds in Belfast, and office-bearers were appointed. 